\title{
Téoros
}

Revue de recherche en tourisme

\section{La gestion des grands fleuves}

Management of the Great Rivers

\section{Michel Lamontagne}

Volume 11, numéro 3, octobre 1992

Fleuves, civilisations et tourisme

URI : https://id.erudit.org/iderudit/1078044ar

DOI : https://doi.org/10.7202/1078044ar

Aller au sommaire du numéro

Éditeur(s)

Université du Québec à Montréal

ISSN

0712-8657 (imprimé)

1923-2705 (numérique)

Découvrir la revue

Citer cet article

Lamontagne, M. (1992). La gestion des grands fleuves. Téoros, 11(3), 42-44. https://doi.org/10.7202/1078044ar d'utilisation que vous pouvez consulter en ligne.

https://apropos.erudit.org/fr/usagers/politique-dutilisation/ 


\title{
La gestion des grands fleuves
}

\author{
Michel Lamontagne*
}

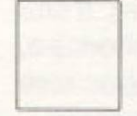

Le thème de cet atelier du colloque Fleuveset planète porte sur la gestion des grands fleuves. Discourir sur ce sujet, à la veille du sommet de Rio, suscite beaucoup de questions pour lesquelles il existe peu de réponses concrètes.

Les discours abondent mais les propositions ne sont pas uniformes. Nous avons peut-être perdu de vue les raisons premières de ces rencontres, et surtout l'objet premier de nos discussions: la sauvegarde pour les générations actuelles et futures d'une eau en quantité suffisante et de bonne qualité tout en maintenant l'équilibre des écosystèmes. Il y a lieu de noter que pratiquement chaque élément d'un écosystème donné est à présent exploitế parl'hommeà des finséconomiques, sociales ou esthétiques.

Afin de replacer le discours dans ses orientations fondamentalementenvironnementales, je m'inspirerai ici d'un document de la Commission économique européenne ainsi que de l'exemple du Plan d'action SaintLaurent (PASL) et je reprendrai certains des principes qui y sont mis de l'avant pour lancer le débat sur une définition pratique de ce concept et sur l'opérationalisation de la gestion écosystémique.

\section{La gestion écosystémique des grands fleuves"'}

Encore aujourd'hui, la notion de gestion écosystémiqueestdébattue àtravers le monde. Les principes opérationnels de ce concept ne sont que partiellement formulés, ce qui fait que peu de pays les ont appliqués.

Le concept de gestion écosystémique offre une perspective intégrée de la planification, de la recherche et de la gestion en matière de ressources en eau qui tient compte non seulement de la durabilité de ces ressources, mais aussi de l'environnement dans son ensemble.

Avant d'opérationaliser de façon concrète cette définition du concept, nous devons tenir compte des principes du développement durable afin de rendre les activités économiques compatibles avec le fonctionnement naturel des écosystèmes. Il vade soi qu'il faut favoriser l'utilisation durable de la ressource eau si l $\mathrm{l}^{7}$ on veut que les besoins des générations actuelles et futures soient satisfaits aussi bien pour ce qui est de la quantité que de la qualité, et ce, tout en maintenant l'équilibre et la qualité de tout écosystème.

Parler de gestion écosystémique implique évidemment lanotion d'écosystème, laquelle englobe:

- l'existence spatiale et temporelle;

- l'intégrité;

- l'ensemble des ressources;

- l'aptitude de l'écosystème à évoluer,

- latolérance et l'adaptabilité (jusqu'àun certain point) à l'évolution des conditions écologiques.

(Par ces deux derniers points, on comprend pourquoi l'opérationalisation du concept de gestion est difficile à réaliser pour des structures politiques comme les nôtres). Les caractéristiques d'un écosystème sont généralement déterminées par l'interaction entre ses éléments.

L'approche de gestion écosystémique suppose également un ensemble intégré de politiques et de pratiques de gestion qui relient l'homme aux écosystèmes dont il fait partie. Cela nécessite par conséquent:

- une synthèse des connaissances integrées;

- une perspective globale de systèmes reliés les uns aux autres à différents niveaux d'intégration;

- desactionsécologiques, prévisionnelles, etc.

D'autre part, les objectifs de la gestion classique de l'eau demeurent des éléments essentiels à la gestion écosystémique des grands fleuves. Ce sont des objectifs qui concernent:

a) la régulation et la distribution des ressources en eau dans le temps et l'espace;
Monsieur M chel Lamontagne est Directeur ex tif, Centre Saint-Laurent, Environnement Canada, Montréal, 
b) l'approvisionnement en eau pour des utilisations diverses;

c) la facilitation du transport des marchandises;

d) la collecte et le traitement des eaux usées;

e) le contrôle des crues.

Tout récemment, cette pratique de gestion s'est enrichie de trois objectifs supplémentaires, à savoir:

f) maintenir l'équilibre écologique de la nature;

g) améliorer ou reconstituer les paysages;

h) protéger ou remettre en état de vastes ensembles naturels dont l'eau est un élément vital.

Somme toute, l'incorporation de considerations liées aux écosystèmes amorce une tentative de définition de principes opérationnels de l'approche écosystémique de gestion qui devrait prévoir:

- des processus de prise de décisions interdisciplinaires concernant l'utilisation futuredes ressourceseneau, compte tenu a la fois de la demande sectorielle et des problèmes d'écosystèmes;

- la recherche de solutions par rapport aux limites des écosystèmes, celles-ci ne correspondant pas nécessairement aux limites administratives ou politiques;

- l'introductiond' une visionà long tenne, celle-ci dépassant l'horizon actuel de la planification économique;

- lagestiondesressources renouvelables;

- la reconstitution des écosystèmes;

- la prévention et le contrôle de la pollution, principalement à la source;

- la préservation de la diversité biologique;

- l'harmonisation des méthodes et des approches;

- la diffusion de l'information aux gestionnaires de l'eau et de l'utilisation des sols, parallèlement à l'éducation du public et à la participation de ce demier à la planification et à la prise de décision;

- l'inscription de matières relatives aux écosystèmes dans l'enseignement universitaire.

Cependant, lesstructures politiques existantes ont été établies pour atteindre des objectifs pointus, sectoriels et sont gérées par différents niveaux d'administration tant national, provincial que local. À l'origine, cette fragmentation correspondaitd' assezprèsaux exigences de gestion. Aujourd' hui, ce n'est plus nécessairement le cas. La CEE propose deux structures reconnues comme essentielles à la bonne marche d'une gestion écosystếmique:

a) l'administration de l'utilisation del'eau et de la protection de l'environnement devrait être assurée par un seul service qui oeuvrerait à une perception commune des objectifs;

b) la participation locale est une condition préalableăuneapprocheécosystémique réaliste.

D'un point de vue théorique, nous constatons que l'approche de gestion écosystémique semble complexe. Cependant, certains pays tentent actuellement d'adapter ces critères à leur mode de gestion de l'environnement.

\section{Le plan d'action Saint-Laurent}

Au Canada, I'approche de gestion écosystémique est l'un des éléments-clés du fonctionnement du Plan d'action SaintLaurent, une initiative du gouvernement canadien pour protéger et conserver le fleuve Saint-Laurent.

Rappelons brièvement les objectifs du Plan d'action Saint-Laurent:

- réduire de $90 \%$ l"ensemble des rejets liquides toxiques déversés dans le fleuve Saint-Laurent par les 50 établissements industriels reconnus comme les plus polluants;

- préparer des plans de restauration de certains milieux contaminés qui sont sous la juridiction du gouvernement fédéral et rếhabiliter les milieux humides perturbés;

- élaborer et mettre en oeuvre des plans de survie pour les espèces menaćes;

- protéger les habitats et créer un parc marin;

- déterminer l'état de l'environnement du fleuve Saint-Laurent.

Ce dernier objectif est l'un des principaux mandats du Centre Saint-Laurent. Concrètement, le Centre Saint-Laurent mène une action soutenue en matière de technologies environnementales, d'écotoxicologie et de chimie organique, de gestion des eaux ainsi que d'évaluation de l'état de l'environnement.
Ses mandats sont:

- la mise sur pied d'un système de suivi de la qualité des écosystèmes;

- la réduction des apports toxiques;

- la mise au point de technologies d'assainissement et de restauration;

- le développement d'une expertise en gestion des grands fleuves;

- la production d'un portrait détaillé de l'environnement du Saint-Laurent.

La réalisation de ces mandats repose essentiellement sur l'établissement de partenariats fuctueux avec les autres paliers de gouvernements, les secteurs privé et universitaire. C'est au Centre Saint-Laurent (CSL) que revient le mandat de gérer les activités scientifiqueset techniques duPASL, de coordonner les efforts de partenariat et d'intégrer les résultats de l'effort collectif.

\section{La gestion écosystémique au Centre Saint-Laurent}

Au CSL, nous croyons que le succès d'une approche de gestion écosystémique repose sur quatre facteurs stratégiquesm:

- la capacité d'intégrer l'information et les données environnementales provenant de différentes sources;

- lacompréhension des relations entre les différents éléments des écosystèmes;

- l'identification de solutions réalistes à des problèmes complexes;

- l'engagement constant de tous les intervenants concernés dans les différents niveaux d'intervention.

Lemandat d'améliorer la connaissance sur le Saint-Laurentetl'obligationderendrecompte de l'état de l'environnement du fleuve sont leséléments qui nous permettentd' appliquer concrètement les principes de gestion écosystémique du fleuve.

Dans ce demier ordre d'idées, le Centre participe activement a lamise enoeuvre d'un programmed'analyseetd'évaluation del'état global de l'environnement. II s'agit d'une entreprise sans préccédent qui consiste à compiler, à synthétiser et à rendre accessible aux intervenants et au grand public l'ensemble des connaissances acquises relativement à l'état de santé du fleuve, afin de se donner des outils pour informer et sensibiliser la population, pour orienter les décisions et développer ici une expertise unique dans la gestion des grands fleuves. 
Cette approche, appelée approche-BILAN, fournit une vision globale du fleuve en utilisant un nouveau cadre conceptuel, qui favorise la diffusion del' information sur le SaintLaurent, au fur et à mesure de l'acquisition des connaissances. Ainsides publications de formats divers s'adressent aux différents publics visés: des feuillets d'information, un atlas environnemental, un système informatisé d'aide à la décision, des rapports techniques, etc. Cette démarche oriente donc la collecte d'informations et le développement d'indicateurs adéquats pour le suivi environnemental du fleuve. Le bilan sur l'état de l'environnement devient un outil essentielă lacomprehensionde l'écosystème, à l'identification de solutions réalistes et sert depoint deréférencepour unir les intervenants dans l'action.

Ce qui différencie la démarche du Centre Saint-Laurent par rapport à d'autres centres de recherche, c'est sa vision intégratrice de l'information à differentes echelles. L'approche des zones d'intérêt prioritaire (ZIP) foumit une vision du fleuve à l'échelle locale. Les ZIP ont pour but de découper le fleuveenplusieurszones pour faciliter l'étude de phénomènes complexes, notamment en matière de diagnostic de la qualité du milieu et des ressources, et pour susciter à l'échelle locale la participation active des partenaires riverains. Les résultats obtenus à ce niveau $\mathrm{d}^{+}$analyse peuvent ensuite être mis en relation avec le bilan environnemental et l'identification de priorités a l'échelle régionale ou même planétaire (figure 1).

Afin d'enraciner sa vocation tant à l'échelle locale qu'internationale, le Centre SaintLaurent oeuvre à l'élaboration d'un concept de réseau de connaissances. La population sera donc appelée à participer à l'intervention et a $l^{\prime}$ action environnementale et par voie de conséquence à la connaissance. Le systềme réseau vise à créer une interface entre les citoyens et les travailleurs du fleuve Saint-Laurent tout comme des autres fleuves situés à la grandeur de la terre. Grâce à ce réseau ouvert, le citoyen pourra au même titre que le chercheur enrichir la banque de données. Ce concept repose donc sur une approche nouvelle dans les acquisitions de l'information environnementale, soit le res: pect des differents niveaux d'information.

Tout récemment, le Centre Saint-Laurent s'est doté d'un programme d'éducation relative a l'environnement. Sa mission est de favoriser aupress de la population riveraine et des decideurs une meilleure compréhension de l'état du Saint-Laurent et du processus d'établissement du bilan environnemental dans les buts d'améliorer la prise de décision et d'encourager l' adoption d'une conduiteen harmonie avec la protection du fleuve.

\section{o Conclusion}

Somme toute, les efforts du Centre SaintLaurent dans I'acquisition et le transfert de la connaissance tentent d'abord d'offrir à la collectivitéainsi qu'aux décideurs politiques, economiques et institutionnels, un éclairage scientifique et pratique afin de favoriser de meillenres décisions et rendre accessible en temps réel la concrétisation d'un environnement fluvial sain.

L'utilisation du bilan environnemental comme moteur del'action environnementale à l'échelle du fleuve, et la création des ZIP pour établir des liens entre les différentes structures de gestion régionale, font du PASL un exemple prometteur de l'application des principes de gestion écosystémique dans les limites de sa juridiction.

La gestion écosystémique soulève pourtant de nombreuses questions selon le contexte politique. Laprotection de fleuves traversant plusieurs pays, commele Niger, le Rhin ou le Danube est vraisemblablement plus difficile àréal iserétant donné le nombred'intervenants et de structures politiques à mobiliser.

Comment peut-on utiliser ou modifier les structures en place pour améliorer la gestion des fleuves? Quels sont les criteres de gestion pratiques que l'on pourrait appliquer qui auraient des effets réels sur l'amélioration de l'étatdes fleuves? Comment peut-on joindre les deux modes de gestion classique et écosystémique?

En tant que gestionnaire, l' utilisation rationnelle et durable des grands fleuves est un défi de taille, d'autant plus que chaque fleuve a ses propres défis. Mettre en relief les besoins et trouver les meilleures solutions, compte tenu des demandes des populations concernées et de l'établissement d'une qualité de base certaine pour nos tleuves, nous obligeront à̀ ne pas nous contenter de demi-succès. Une approche de gestion ccosystémique semble l'un des moyens pour atteindre ce but.

Je souhaite que le sommet dit de la dernière chance, a Rio de Janeiro, permette de jeter un éclairage nouveau en matière de gestion de l'eau. Des critères comme le maintien de l'équilibre écologique de la nature, la recherche de solutions dans les limites des écosystèmes, l'informationetl'éducationdes gestionnaires de l'eau etdes sols, doiventêtre pris en compte pour en arriver à une polarisationdes échangesentre gestionnaires et constituer le point de départ vers une nouvelle coopération nationale et internationale $f$

\section{RÉFÉRENCES}

(1) Conseil economique pour l"Europe, L'approche par écosystàme de la gestion de l'eau, séminaire presente a Oslo (Norvege), du 27 au 31 mai 1991.

(2) Nicole Lavigne, Ecosystems Approach to River Basin Management: Integrated SOE Reporting at the Hearth of Concerned Action, communieation presentée a Ronterdam (Pays-Bas), du 18 22 mai 1991.

\section{Management of the Creat Rivers}

\section{By Michel Lamontagne}

This presentation focuses on the theme of the management of the great rivers, and its objective is to launch the debate on a practical definition of the concept of great-river management. Inspiration wil be sought among the elements of ecosystem. based management, and taking existing policy structures and marketing characteristics into consideration.

For example, the EEC proposes a list of operating principles for ecosystembased management such as interdisciplinary decision-making, searching for solutions in relation to ecosystemic boundaries. long-term vision, information dissemination and public education, ete. In addition, the St. Lawrence Action Plan (PASL) is presented as an example of an application of the ecosystem-based management concept. The programme produces records on the environmental status based on four strategicfactors: the capacity to integrate information with environmental data; comprehension of the relationships which exist between variouselements of the ecosystems; identification of realistic solutions; the committment of contributors on all levels of intervention.

Thus, the 5t. Lawrence Centre of Environment Canada, which administers PASL activities of a scientific nature, applies a vision which integrates information on different scales. This innovatwe strategy is based on two complementary approaches: the BILAN approach, which supplies an overall vision of the river and the ZIP (priority interest zones) approach, which views the river on a local scale. Results obtained at these different tevels of analysis allow pricrities to be established on a regional or even planetary level.

These examples are presented to serve as a basis of discussion towards developing a definition of the concept of great-river manacement. These different schools of thought all target the same objective: to ensure a rational and sustainable utilization of the great rivers. 